\title{
Application of MCSFilter to Estimate Stiction Control Valve Parameters
}

\author{
Andreia Amador ${ }^{1, \mathrm{~b})}$, Florbela P. Fernandes ${ }^{2, \mathrm{a})}$, Lino O. Santos ${ }^{1, \mathrm{c})}$ and Andrey \\ Romanenko ${ }^{1,3, \mathrm{~d})}$ \\ ${ }^{1}$ CIEPQPF, Department of Chemical Engineering, University of Coimbra, Portugal. \\ ${ }^{2}$ Polytechnic Institute of Bragança, Portugal. \\ ${ }^{3}$ Ciengis, SA, Portugal. \\ ${ }^{a)}$ Corresponding author: fflor@ipb.pt \\ b) uc2010149251@student.uc.pt \\ c)lino@eq.uc.pt \\ d) andrey.romanenko@ ciengis.com
}

\begin{abstract}
The mitigation of the stiction phenomena in control valves is of paramount importance for efficient industrial plant operation. Mathematical models of sticky valves are typically discontinuous and highly nonlinear. A derivative-free optimization method is applied in the context of parameter estimation in order to determine the stiction parameters of a control valve. The method successfully determines the correct parameter set and compares favorably with a previous case study of this problem that used smooth function.
\end{abstract}

\section{INTRODUCTION}

The optimization methods and tools are of paramount importance in chemical process engineering research and development. In fact, they play a major role in the decision process regarding the implementation and the improvement of optimal process design, operation, and control solutions [1]. In many cases, the mathematical model used to describe the physical behavior of a specific part of the process can present discontinuities and/or severe non-linearities. This may lead to difficulties in obtaining the derivative information of the model. Such situations may be successfully handled by derivative-free methods.

Process control valves are the final control element and the only mechanical part in a process control loop. For this reason, valve performance has a great influence on process productivity, final product quality, and utilities consumption $[2,3]$.

A critical issue associated with control valve malfunction is stiction, which is due to excessive static friction. In general, static friction occurs when there are two solid objects sliding in contact to each other. This is the case of the contact between the case of the packing area and the stem in the control valve. An important point here is that weariness of these valve components intensifies the stiction problem. This makes worse the valve malfunctioning, causing a degradation of the process operation performance and economic losses. This topic has received a great deal of attention from the research community, with the proposal of several modelling approaches to support the development of tools for identification and diagnosis of valve stiction. A thoroughly overview on this topic is available in [4].

The problem of estimating stiction parameters of the dynamic model of a sticky valve constitutes an essential stage within a optimization and control framework of an industrial plant where the performance of the final element controls is explicitly taken into consideration. Very often, these dynamic models, or input/output models are non-differentiable, featuring nonlinearities and/or discontinuities. These features may limit the applicability of optimization methods that are not derivative-free. This is the case of mathematical models proposed to describe a valve with stiction. 
In this work, the derivative-free optimization method MCSFilter [5] is applied in order to determine the valve stiction parameters of the Chen discrete-time model [6] that describes the behavior of a sticky valve after a set of four distinct patterns. It establishes the corresponding physical conditions that lead to switching between the patterns of behavior.

The manuscript is organized as follows. First, a brief description of the MCSFilter method is given. Next, the sticky valve model is presented, and the parameter estimation problem is stated. This is followed by a presentation and discussion of the results obtained in a Monte Carlo simulation. Finally, the main conclusions are drawn and future work directions are pointed out.

\section{MCSFilter METHOD}

The MCSFilter method is able to find multiple solutions of nonconvex and nonlinear constrained optimization problems. Since it does not require any derivative information, it is particularly suitable to problems with discontinuous objective or constraint functions.

The exploration feature of the method is based on a multistart strategy, which is a stochastic algorithm that repeatedly applies a local search to sampled points aiming to converge to all the solutions of a multimodal problem. When this strategy is applied, some or all of the minimizers may be found more than once. To avoid convergence to a previously computed solution, a clustering technique based on computing the regions of attraction of previously identified minimizers is used. Figure 1 illustrate the influence of the regions of attraction. The black line between the initial approximation and the minimizer represents the local search that took place. The white dashed line between the two points represents a local search that was discarded by the regions of attraction.

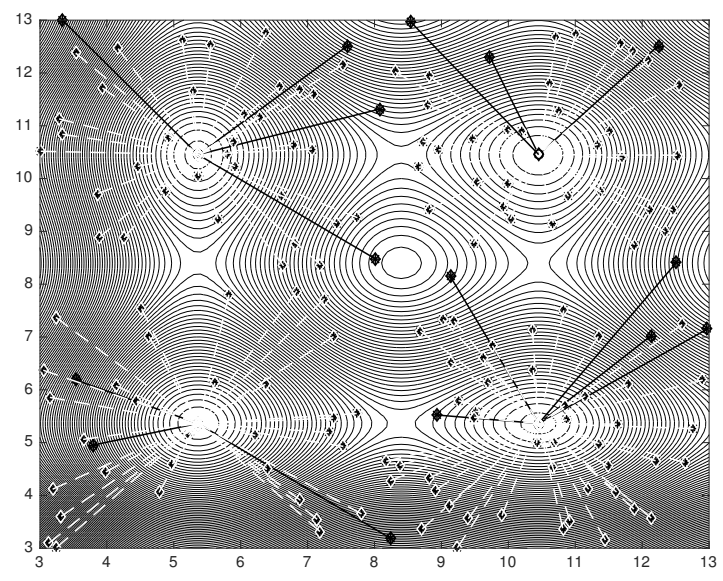

FIGURE 1. Illustration of the multistart algorithm with regions of attraction.

The exploitation of promising regions is made by a derivative-free local search that consists of a coordinate search combined with a filter methodology to generate a sequence of approximate solutions that improve either the constraint violation or the objective function relative to the previous approximation [5].

\section{STICKY VALVE DISCRETE-TIME MODEL}

The sticky valve discrete-time model is based on the Chen model [6], and it can be stated in the following form [3],

$$
z(k)= \begin{cases}z_{1}(k), & \text { if } e_{1}(k)=1 \text { and } e_{2}(k) \geqslant 0, \\ z_{2}(k), & \text { if } e_{1}(k)=1 \text { and } e_{2}(k)<0, \\ z_{3}(k), & \text { if } e_{1}(k)=0 \text { and } e_{3}(k)<0, \\ z_{4}(k), & \text { if } e_{1}(k)=0 \text { and } e_{3}(k) \geqslant 0,\end{cases}
$$


where $z(k)^{\mathrm{T}}=[c(k) x(k) s(k)]$ is the vector of valve outputs at every time instant $k$. The valve can exhibit four patterns of behavior, $i=1, \ldots, 4$. These are indicated in Table 1 with the corresponding definition of $z(k)$. The output variable

TABLE 1. Sticky valve behaviors and the corresponding valve outputs $(z(k)$ in (1)).

\begin{tabular}{llll}
\hline stick to slip & keep sticking & slip to stick & keep slipping \\
$i=1$ & $i=2$ & $i=3$ & $i=4$ \\
\hline$c(k)=d(k) \cdot f_{\mathrm{D}}$ & $c(k)=c(k)$ & $c(k)=c(k)$ & $c\left(\left(k=d(k) \cdot\left|f_{\mathrm{D}}\right|\right.\right.$ \\
$x(k)=u(k)-c(k)$ & $x(k)=x(k-1)$ & $x(k)=x(k-1)$ & $x(k)=u(k)-c(k)$ \\
$s(k)=0$ & $s(k)=s(k-1)$ & $s(k)=1$ & $s(k)=s(k-1)$ \\
\hline
\end{tabular}

$c(k)$ is the accumulated force compensated by friction at time instant $k$, and it is given by

$$
c(k)=c(k-1)+u(k)-u(k-1),
$$

where $u(k)$ is the input signal to the valve. The variable $s(k)$ is the valve status flag, and $e_{i}(k), i=1,2,3$, are given respectively by

$$
e_{1}(k)=s(k-1), \quad e_{2}(k)=c(k)-f_{\mathrm{S}}, \quad e_{3}(k)= \begin{cases}1, & \text { if } f_{\mathrm{D}}>0 \text { and }|c(k)|>f_{\mathrm{D}}, \\ 1, & \text { if } f_{\mathrm{D}}=0 \text { and } d(k) \cdot d(k-1)>0, \\ 1, & \text { if } f_{\mathrm{D}}<0 \text { and }|c(k)|<-f_{\mathrm{D}}, \\ 0, & \text { otherwise }\end{cases}
$$

where $d(k)$ is the actual direction of valve sliding. It is noteworthy that the sticky valve model (1-3) is nonlinear and discontinuous. Therefore, its numerical implementation requires several if-then-else constructs. The static friction, $f_{\mathrm{S}}$, is given by

$$
f_{\mathrm{S}}=f_{\mathrm{D}}+f_{\mathrm{J}},
$$

where $f_{\mathrm{D}}$ is the kinetic friction, and $f_{\mathrm{J}}$ is the slip jump. Hence, the vector of parameters of the sticky valve model (1-3) is $p^{T}=\left[f_{\mathrm{D}} f_{\mathrm{J}}\right]$. Further details on the rationale behind this modeling approach can be found in $[3,6]$.

\section{STICTION VALVE PARAMETER ESTIMATION PROBLEM}

The problem of estimating $p$ is cast as an optimization problem stated as follows,

$$
\begin{array}{cl}
\underset{p}{\operatorname{minimize}} & J=\left(\mathbf{z}_{\exp }-\mathbf{z}\right)^{\mathrm{T}} \mathbf{Q}\left(\mathbf{z}_{\mathrm{exp}}-\mathbf{z}\right), \\
\text { subject to } & \mathbf{z}_{\mathrm{L}} \leqslant \mathbf{z} \leqslant \mathbf{z}_{\mathrm{U}} \\
& \mathbf{u}_{\mathrm{L}} \leqslant \mathbf{u} \leqslant \mathbf{u}_{\mathrm{U}} \\
& p_{\mathrm{L}} \leqslant p \leqslant p_{\mathrm{U}}
\end{array}
$$

where $\mathbf{z}$ is the vector of the valve positions over a given time horizon with $n$ sampling time points, and $\mathbf{u}$ is the corresponding vector of valve inputs. The quadratic objective function (5a) is defined in terms of the difference between the experimental output profile $\mathbf{z}_{\mathrm{exp}}$ and the model prediction profile $\mathbf{z} . \mathbf{Q}$ is a diagonal weighting matrix. Lower and upper bounds on the output and input variables over the time horizon, and on the model parameters are defined in $(5 b-5 d)$.

The valve positions are determined using the discrete-time model (1-3) that can be presented in a compact form as $\mathbf{z}=\mathrm{f}\left(\mathbf{z}_{(\mathrm{k}-1)}, \mathbf{u}, p\right)$, where the notation $\mathbf{z}_{(\mathrm{k}-1)}$ stands for the vector $\mathbf{z}$ with the elements one time instant shifted backward.

\section{RESULTS AND DISCUSSION}

Problem (5) is solved using the derivative-free method MCSFilter. To illustrate this application, the vectors $\mathbf{z}_{\exp }$ and $\mathbf{u}$ are defined with data obtained by simulation from [3], using $f_{\mathrm{D}}=0.90$ and $f_{\mathrm{J}}=1.90$. The number of sampling time 
points is $n=98$. The initial guess for the parameters is $f_{\mathrm{D}}=f_{\mathrm{J}}=0.20$. The following bounds are used: $0 \leqslant f_{\mathrm{D}} \leqslant 5$, $0 \leqslant f_{\mathrm{J}} \leqslant 5,0 \leqslant x(k) \leqslant 1$ and $0 \leqslant u(k) \leqslant 1, k=1, \ldots, n$. The weighting matrix in the cost function is set to the identity matrix. A Monte Carlo simulation with 1000 runs was performed. In each run the optimization problem (5) is solved with a randomly chosen starting point in the parameter space. This numerical application was implemented in the Java programming language. The average runtime to solve the optimization problem is $7 \times 10^{-2} \mathrm{~s}$ on a $2,6 \mathrm{GHz}$ laptop with the MacOS El Capitan operating system. The results of the Monte Carlo study are summarized in Table 2.

TABLE 2. Optimal stiction parameters and cost function.

\begin{tabular}{ccccc}
\hline & Best & Worst & Average & {$[3]$} \\
\hline$J^{*}$ & $6.19 \times 10^{-11}$ & $1.55 \times 10^{-2}$ & $4.18 \times 10^{-5}$ & $1.00 \times 10^{-3}$ \\
$f_{\mathrm{J}}^{*}$ & 1.868 & 1.700 & - & 1.732 \\
$f_{\mathrm{D}}^{*}$ & 0.899 & 0.913 & - & 0.898 \\
\hline
\end{tabular}

The best optimal solution (Table 2) obtained with the derivative-free MCSFilter method compares favorably with the optimal solution obtained solving the optimization problem with a SQP approach applied to a smoothed model (last column of Table 2). The best value of the objective function is much lower than the one present in [3], and the estimated parameters are very close to the real values $\left(f_{\mathrm{D}}=0.90\right.$ and $\left.f_{\mathrm{J}}=1.90\right)$.

\section{CONCLUSIONS AND FUTURE WORK}

The MCSFilter method was implemented in Java and applied to determine the stiction parameters of the Chen model of a sticky control valve. The results, of the case study show that the optimal solution is closer to the real values when compared to the optimal solution obtained using an SQP method and a smooth function in a previous work. Furthermore, these results show that a derivative-free method may achieve better solution in problems presenting discontinuities or the lack of derivative information.

Although, the results obtained herein are promising, a further comparative study between MCSFilter and other well known derivative-free optimization methods will be carried out.

\section{REFERENCES}

[1] T. G. Kolda, R. M. Lewis and V. Torczon, Siam Rev. 45, 385-482 (2003)

[2] M. A. A. S. Choudhury, S. L. Shah and N. F. Thornhill, Diagnosis of Process Nonlinearities and Valve Stiction (Springer-Verlag, Berlin, 2008).

[3] A. S. R. Brásio, A. Romanenko and N. C. P. Fernandes, "Stiction detection and quantification as an application of optimization", in ICCSA 2014, Part II, Lect. Notes Comput. Sc. 8580, edited by B. Murgante et al. (Springer International Publishing, Switzerland, 2014) 169-179.

[4] A. S. R. Brásio, A. Romanenko and N. C. P. Fernandes, Ind. Eng. Chem. Res. 53, 15020-15040 (2014).

[5] F. P. Fernandes, M. F. P. Costa, and E. M. G. P. Fernandes, "Multilocal Programming: a Derivative-Free Filter Multistart Algorithm", in ICCSA 2013, Part I, Lect. Notes Comput. Sc. 7971, edited by B. Murgante et al. (Springer, Berlin Heidelberg, 2013) 333-346.

[6] S.-L. Chen, K. K. Tan and S. Huang, Ind. Eng. Chem. Res. 47(8), 2842-2848 (2008). 